TRANSACTIONS OF THE

AMERICAN MATHEMATICAL SOCIETY

Volume 362, Number 8, August 2010, Pages 4243-4265

S 0002-9947(10)05002-6

Article electronically published on March 23, 2010

\title{
DEPTH ZERO BOOLEAN ALGEBRAS
}

\author{
ASHER M. KACH
}

\begin{abstract}
We study the class of depth zero Boolean algebras, both from a classical viewpoint and an effective viewpoint. In particular, we provide an algebraic characterization, constructing an explicit measure for each depth zero Boolean algebra and demonstrating there are no others, and an effective characterization, providing a necessary and sufficient condition for a depth zero Boolean algebra of rank at most $\omega$ to have a computable presentation.
\end{abstract}

\section{INTRODUCTION}

A fundamental problem in the field of algebra is to classify the isomorphism types existing within a class of structures. If a class of countable structures is sufficiently well understood classically, questions of effectiveness may also be addressed. Chief among them is: Which isomorphism types have computable presentations? Roughly speaking, a computable presentation is a finite description of the algebraic structure's universe and the relations and operations on it.

For several naturally occurring classes of algebraic structures, the classical and computable characterization is straightforward. Countable vector spaces over the rational numbers (or any field) and countable algebraically closed fields of characteristic zero, for example, are both characterized classically by a single cardinal number (their dimension or transcendence degree, respectively) and always have computable presentations (provided the underlying field is computable in the case of vector spaces).

Other natural classes have more complex classical and computable characterizations. Countable reduced abelian $p$-groups, for example, are characterized classically by Ulm invariants, countable sequences (not necessarily of order type $\omega$ ) of countable cardinals (see [6] or [10, for example). Amongst reduced abelian $p$ groups of height less than $\omega^{2}$, an isomorphism type is computable if and only if the Ulm invariants can be specified by a finite number of limitwise monotonic functions over appropriate oracles (see 9]).

Vastly more complicated is the subject of this paper, Boolean algebras. Ketonen (see [11]) found natural invariants that characterize the isomorphism type of countable Boolean algebras. Here we study the Boolean algebras with the simplest invariants, the depth zero Boolean algebras. Classically, we add a constructive

Received by the editors June 9, 2008.

2000 Mathematics Subject Classification. Primary 03D45.

Key words and phrases. Boolean algebras, Ketonen invariants, depth zero.

The author thanks his thesis advisor, Steffen Lempp, for all his guidance and suggestions; Christopher Alfeld, Robert Owen, and Daniel Turetsky for numerous conversations, comments, and corrections; and the anonymous referee for his/her comments.

(C)2010 American Mathematical Society Reverts to public domain 28 years from publication 
characterization of them (Section 3) to the algebraic characterization offered by Pierce (see [13]). Effectively, we provide a necessary and sufficient condition for a rank $\omega$ depth zero Boolean algebra to have a computable presentation (Section 4). We note that this deviates from the historical approach at attempting to answer which Boolean algebras have computable presentations. Previous attempts have centered on the program of showing that if a Boolean algebra has a presentation of small complexity, then it has a computable presentation (see [4, [12, and [18]). Though the historical approach has not been entirely successful, as a corollary to our approach we obtain the desired historical result for rank $\omega$ depth zero Boolean algebras (Section 5).

As the reader is not assumed to be familiar with Ketonen invariants, we include a brief, self-contained review of these invariants for depth zero Boolean algebras (Section 2).

\section{BACKGROUND AND NOTATION}

Although a thorough discussion of Ketonen invariants is beyond the scope of this paper (see [11 for the original paper or [13] for an alternative exposition), we do discuss the background necessary to understand the depth zero Boolean algebras. Before doing so, we begin with the following important convention.

Convention 2.1. Throughout, our Boolean algebras and our linear orders are all assumed to be countable.

We also freely use the set-theoretic convention of using an ordinal to denote the set of ordinals below it, i.e., using $\alpha$ to denote the set of ordinals $\beta$ less than $\alpha$.

We view Boolean algebras as structures in the signature $\mathcal{B}=(B:+, \cdot,-, 0,1)$. We use the notation $x \oplus y$ to denote the element $x+y$ with the additional hypothesis that $x y=0$. As we will frequently have the need to use the symbols 0 and 1 to denote ordinals, we will always refer to the largest and smallest elements of a Boolean algebra $\mathcal{B}$ as $1_{\mathcal{B}}$ and $0_{\mathcal{B}}$.

As describing (a construction of) a fixed Boolean algebra $\mathcal{B}$ can often be quite cumbersome, we will often describe (a construction of) a linear order $\mathcal{L}$ whose interval algebra is isomorphic to $\mathcal{B}$.

Definition 2.2. Let $\mathcal{L}$ be a linear order with least element $x_{0}$ with the topology generated by basic open sets $[a, b)$. The interval algebra of $\mathcal{L}$, denoted $\mathcal{B}_{\mathcal{L}}$, is the Boolean algebra whose universe is the set of clopen subsets of $\mathcal{L}$. The operations of join, meet, and complementation in the Boolean algebra $\mathcal{B}_{\mathcal{L}}$ are given by taking the union, intersection, and complementation of the clopen sets.

If $\mathcal{B}$ is a Boolean algebra, then $\mathcal{L}$ is a linear order generating $\mathcal{B}$ if $\mathcal{B} \cong \mathcal{B}_{\mathcal{L}}$.

An important fact is that both directions of the Stone Representation Theorem, i.e., the transitions from a Boolean algebra $\mathcal{B}$ to a linear order $\mathcal{L}_{\mathcal{B}}$ that generates it, and from a linear order $\mathcal{L}$ to its interval algebra $\mathcal{B}_{\mathcal{L}}$, are effective. We emphasize that the linear order $\mathcal{L}_{\mathcal{B}}$ need not be unique, and, in most cases, is actually far from unique. However, by applying certain other algebraic manipulations to $\mathcal{B}$, a unique invariant can be obtained. Our approach combines those of Heindorf and Pierce (see [8] and [13]).

Definition 2.3. If $\mathcal{B}$ is any Boolean algebra, denote by $\mathrm{SA}(\mathcal{B})$ the set of superatomic elements of $\mathcal{B}$. Recall that an element is superatomic if and only if it is isomorphic to the interval algebra of an ordinal. 
Definition 2.4. If $\mathcal{B}$ is any Boolean algebra and $x \in \mathcal{B}$ is nonzero superatomic, the $\operatorname{rank}$ of $x$, denoted $\operatorname{rank}(x)$, is the ordinal $\alpha+1$ for which there is an integer $n$ with $x \cong \operatorname{Int} A \lg \left(\omega^{\alpha} \cdot n\right)$; the rank of the zero element $0_{\mathcal{B}}$ is zero.

If $\mathcal{B}$ is any Boolean algebra and $x \in \mathcal{B}$ is not superatomic, the rank of $x$, denoted $\mu_{\mathcal{B}}(x)$, is given by

$$
\mu_{\mathcal{B}}(x)=\sup \{\operatorname{rank}(y): y \leq x, y \in \mathrm{SA}(\mathcal{B})\} .
$$

Definition 2.5. If $\mathcal{B}$ is any Boolean algebra, define its measure (with domain $\mathcal{B}$ ) to be the function $\hat{\sigma}_{\mathcal{B}}: \mathcal{B} \rightarrow \omega_{1} \cup\{o\}$ given by

$$
\hat{\sigma}_{\mathcal{B}}(x)= \begin{cases}o & \text { if } x \in \operatorname{SA}(\mathcal{B}), \\ \min \left\{\mu_{\mathcal{B}}(y): y \leq x, x-y \in \operatorname{SA}(\mathcal{B})\right\} & \text { otherwise }\end{cases}
$$

Here $o$ is a special symbol that (by definition) satisfies $o<\alpha$ for any ordinal $\alpha \in \omega_{1}$.

The reader is cautioned that the choice of terminology is slightly misleading; these measures have no real connection to the measures found in analysis.

The motivation for the definition of $\hat{\sigma}_{\mathcal{B}}$ arises since it is undesirable that the inequality $\mu_{\mathcal{B}}(x+y)>\mu_{\mathcal{B}}(x)$ can happen when $y \in \mathrm{SA}(\mathcal{B})$. We assign a special name for Boolean algebras in which this phenomenon doesn't happen.

Definition 2.6. A Boolean algebra $\mathcal{B}$ is uniform if $\mu_{\mathcal{B}}\left(1_{\mathcal{B}}\right)=\mu_{\mathcal{B}}\left(1_{\mathcal{B}}-x\right)$ for all $x \in \mathrm{SA}(\mathcal{B})$.

Restricting one's attention to only the uniform Boolean algebras does no harm (classically or effectively) as the following proposition (see [11]) and corollary demonstrate in conjunction with the superatomic Boolean algebras being well understood (classically and effectively).

Proposition 2.7. Every Boolean algebra $\mathcal{B}$ is the disjoint sum of a uniform Boolean algebra $\mathcal{B}_{u}$ and a superatomic Boolean algebra $\mathcal{B}_{s}$.

Before showing that $\mathcal{B}_{u}$ and $\mathcal{B}_{s}$ are computable if $\mathcal{B}$ is computable, we give three illustrative examples of Proposition 2.7.

Example 2.8. If $\mathcal{B}=\operatorname{Int} A \lg (n+\eta)$, then $\mathcal{B}_{u} \cong \operatorname{IntAlg}(1+\eta)$ and $\mathcal{B}_{s} \cong \operatorname{Int} A \lg (n)$.

If $\mathcal{B}=\operatorname{Int} \operatorname{Alg}\left(\omega^{\alpha} \cdot(1+\eta)\right)$, then $\mathcal{B}_{u} \cong \operatorname{Int} \operatorname{Alg}\left(\omega^{\alpha} \cdot(1+\eta)\right)$ and $\mathcal{B}_{s}$ is trivial.

If $\mathcal{B}=\operatorname{IntAlg}\left(\omega^{\beta}+\omega^{\alpha} \cdot(1+\eta)\right)$ with $\beta>\alpha$, then $\mathcal{B}_{u} \cong \operatorname{IntAlg}\left(\omega^{\alpha} \cdot(1+\eta)\right)$ and $\mathcal{B}_{s} \cong \operatorname{IntAlg}\left(\omega^{\beta}+1\right)$.

Corollary 2.9. Every computable Boolean algebra is the disjoint sum of a computable uniform Boolean algebra and a computable superatomic Boolean algebra.

Proof. Let $\mathcal{B}$ be a computable Boolean algebra. From Proposition 2.7 we obtain a classical decomposition $\mathcal{B} \cong \mathcal{B}_{u} \oplus \mathcal{B}_{s}$ with $\mathcal{B}_{u}$ uniform and $\mathcal{B}_{s}$ superatomic. The isomorphism type of $\mathcal{B}_{s}$ must be computable as a consequence of $\mathcal{B}$ being computable.

Fix a computable presentation of $\mathcal{B}$. If $x$ is any element of $\mathcal{B}$ whose subalgebra is isomorphic to $\mathcal{B}_{s}$, then $\mathcal{B}_{u} \cong \mathcal{B}-x$. As both $x$ (as a subalgebra of $\mathcal{B}$ ) and $\mathcal{B}$ are computable, the subalgebra $\mathcal{B}_{u}$ is computable (indeed, as a subalgebra of $\mathcal{B}$ ).

It becomes cumbersome to work with the function $\hat{\sigma}_{\mathcal{B}}$ as defined, and it is in fact somewhat unnecessary to do so. Beyond domain information, the definition of $\hat{\sigma}_{\mathcal{B}}$ on superatomic elements provides no information. We therefore seek a function defined on the free Boolean algebra. 
Definition 2.10. The free Boolean algebra $\mathcal{F}$ (on countably many generators), alternately the (countable) atomless Boolean algebra, is the (unique) nontrivial Boolean algebra having no atoms.

We view $\mathcal{F}$ as being the Boolean algebra generated by the set of strings $\tau \in 2^{<\omega}$, i.e., elements are finite unions of these cones.

The observation that $\mathcal{F}$ is isomorphic to the quotient of any nonsuperatomic Boolean algebra $\mathcal{B}$ by the ideal $\mathcal{I}$ generated by its superatomic elements allows us to simplify the domain of a measure (with domain $\mathcal{B}$ ) to $\mathcal{F}$.

Definition 2.11. If $\mathcal{B}$ is any Boolean algebra and $\pi: \mathcal{B} / \mathcal{I} \rightarrow \mathcal{F}$ is an isomorphism, define its measure (with respect to $\pi$ ) to be the function $\varsigma_{\mathcal{B}}: \mathcal{F} \rightarrow \omega_{1} \cup\{o\}$ given by

$$
\varsigma_{\mathcal{B}}(x)=\hat{\sigma}_{\mathcal{B}}(z)
$$

for any $z \in \mathcal{B}$ such that $\pi([z])=x$.

We note that $\varsigma_{\mathcal{B}}$ is well defined for if $\pi([z])=\pi\left(\left[z^{\prime}\right]\right)$, then $z$ and $z^{\prime}$ differ by at most a superatomic element, and consequently $\hat{\sigma}_{\mathcal{B}}(z)=\hat{\sigma}_{\mathcal{B}}\left(z^{\prime}\right)$.

The choice of the isomorphism $\pi$ is unimportant, so we will omit any reference to it. Because of the natural embedding $\iota$ of $2^{<\omega}$ into $\mathcal{F}$, we can further simplify the domain of a measure.

Definition 2.12. If $\mathcal{B}$ is any Boolean algebra, define its measure (with domain $2^{<\omega}$ ) to be the function $\sigma_{\mathcal{B}}: 2^{<\omega} \rightarrow \omega_{1}$ given by

$$
\sigma_{\mathcal{B}}(x)=\varsigma_{\mathcal{B}}(\iota(x)) .
$$

Conversely, if $\sigma: 2^{<\omega} \rightarrow \omega_{1}$ is any map satisfying $\sigma(\tau)=\max \left\{\sigma\left(\tau^{\frown} 0\right), \sigma\left(\tau^{\frown} 1\right)\right\}$ for all $\tau \in 2^{<\omega}$, define a map $\varsigma_{\sigma}: \mathcal{F} \rightarrow \omega_{1} \cup\{o\}$ by

$$
\varsigma_{\sigma}(x)=\sup \left\{\sigma(\tau): \tau \in 2^{<\omega}, \iota(\tau) \leq x\right\}
$$

and $\varsigma_{\sigma}\left(0_{\mathcal{F}}\right)=o$. Let $\mathcal{B}=\mathcal{B}_{\varsigma}$ be the (unique) Boolean algebra having $\varsigma$ as its measure.

A measure $\sigma$ can thus be viewed as an ordinal-labeled complete binary branching tree $T_{\sigma}$, where the label at a node $\tau$ in the tree is $\sigma(\tau)$.

The three definitions (Definition 2.5, Definition 2.11, and Definition 2.12) should not be thought of as separate definitions of measures, but rather three ways to view the same algebraic object. Regardless of the interpretation used, when we speak of the range of a measure $\sigma$, we mean the set of ordinals appearing in the range of $\sigma$. Note that it does no harm to ignore the special element $o$ as it appears in the range of every measure.

In order to help prevent confusion, we reserve the symbol $\sigma$ exclusively for measures (with the domain making it clear which interpretation is being used) and the symbol $\tau$ exclusively for binary strings. In order to avoid cumbersome language, we make little or no distinction between $\sigma, \varsigma$, and $T_{\sigma}$. We also make little distinction between a measure $\sigma$, the Boolean algebra $\mathcal{B}_{\sigma}$, and any linear order $\mathcal{L}$ that generates $\mathcal{B}_{\sigma}$. As the context will always make the meaning transparent, there should be no confusion.

We end our discussion of Ketonen invariants by introducing depth zero Boolean algebras. 
Definition 2.13. If $\sigma: \mathcal{F} \rightarrow \omega_{1} \cup\{o\}$ is a measure, define its derivative to be the map $\Delta \sigma: \mathcal{F} \rightarrow \mathcal{P}\left(\left[\omega_{1} \cup\{o\}\right]^{<\omega}\right)$ given by

$$
\Delta \sigma(x)=\left\{\left(\sigma\left(x_{1}\right), \ldots, \sigma\left(x_{n}\right)\right): x=x_{1} \oplus \cdots \oplus x_{n}\right\} .
$$

Definition 2.14. A Boolean algebra $\mathcal{B}=\mathcal{B}_{\sigma}$ is depth zero if

$$
\forall x \forall y[\sigma(x)=\sigma(y) \Longrightarrow \Delta \sigma(x)=\Delta \sigma(y)] .
$$

A fundamental result by Ketonen (which he generalized greatly) is that the derivative $\Delta \sigma\left(1_{\mathcal{F}}\right)$ characterizes the isomorphism type of $\mathcal{B}_{\sigma}$ amongst the depth zero Boolean algebras.

Although we end our discussion of Ketonen invariants here, the reader is referred to [11] or [13] for a more thorough exposition and a discussion of higher depth Boolean algebras or to 3 for a discussion of the complexity of the isomorphism relation for Boolean algebras. The reader is also referred to [7] or 14 for an exposition of Ershov-Tarski invariants. These invariants (which are far simpler) characterize the elementary equivalence classes of the countable Boolean algebras rather than their isomorphism classes, as Ketonen invariants do.

Before moving to studying the depth zero Boolean algebras, we introduce the Feiner hierarchy. In [5], Feiner defined a hierarchy of complexities for certain sets $S$ computable in $\emptyset^{(\omega)}$. The idea was that for some sets $S$ computable in $\emptyset^{(\omega)}$, there are integers $a$ and $b$ such that membership of $n \in S$ could be computed uniformly from the oracle $\emptyset^{(b n+a)}$. The reader is assumed to be familiar with fundamental notions of computability theory and is referred to [15] or [16] for such a background.

In this context, we need membership of $n \in S$ to be an existential question over the oracle $\emptyset^{(2 n+2)}$. We therefore use the following definition.

Definition 2.15. A set $S \subseteq \omega$ is $\Sigma_{(2 n+3)}^{0}$ if there is an index $e$ such that for all $n$, $n \in S$ if and only if $\varphi_{e}^{\emptyset^{(2 n+2)}}(n) \downarrow$.

\section{DePth Zero MEASURES}

In this section we construct, for every set $S \subseteq \omega_{1}$ with greatest element, two depth zero measures with range $S$ (Section 3.1) and demonstrate that there are no other depth zero measures with range $S$ (Section 3.2). Before demonstrating this, we pause to note that it should not be surprising that there are so few depth zero measures. For if $\sigma$ is depth zero, by definition $\sigma(x)=\sigma(y)$ implies $\Delta \sigma(x)=\Delta \sigma(y)$. Thus the isomorphism type of an element $x$ within a depth zero measure $\sigma$ is totally determined by $\sigma(x)$. Knowing the range of $\sigma_{\mathcal{B}}$ is thus almost enough to identify $\mathcal{B}$. The only information missing, as we shall see, is whether there exist disjoint elements $x$ and $y$ with $\sigma(x)=\max (S)=\sigma(y)$.

Though this is the first constructive characterization of the depth zero measures, other characterizations of the depth zero measures are already known. Pierce, for example, provided an algebraic characterization for a set $\Delta \sigma\left(1_{\mathcal{F}}\right)$ to be the first derivative of a depth zero measure using the Local Refinement Property (see Proposition 1.18.5 of [13]). Heindorf (see Lemma 5.2 of [8]) also provided an explicit characterization of the first derivatives of depth zero measures (as every depth zero measure is pseudo-indecomposable and primitive; see Theorem 3.8). We contribute a constructive description of these measures, a necessary component for an analysis of which ones have computable presentations in addition to being useful in their own right. 
As preparation, we define certain strings $\tau \in 2^{<\omega}$ to be repeater strings, almost repeater strings, and xor strings.

Definition 3.1. A string $\tau \in 2^{<\omega}$ is a repeater string if the length $|\tau|$ of $\tau$ is even and $\tau(2 i)=\tau(2 i+1)$ for all $i<|\tau| / 2$.

A string $\tau \in 2^{<\omega}$ is an almost repeater string if $\tau$ is a repeater string or of the form $\tau=\tau^{\prime} \frown 0$ or $\tau=\tau^{\prime} \frown 1$ for some repeater string $\tau^{\prime}$.

A string $\tau \in 2^{<\omega}$ is an xor string if either $\tau=01$ or $\tau=10$.

3.1. Existence. We demonstrate the existence of two depth zero measures with range $S$, termed $\sigma_{u(S)}$ and $\sigma_{v(S)}$, for each set $S \subseteq \omega_{1}$ with greatest element. We begin by showing the existence of $\sigma_{u(S)}$ and $\sigma_{v(S)}$ in the special case when $S$ is a nonzero ordinal. In the special case when $S=1$, the measures $\sigma_{u(1)}$ and $\sigma_{v(1)}$ coincide.

Lemma 3.2. There is a depth zero measure with range $1=\{0\}$.

Proof. Let $\sigma_{u(1)}$ and $\sigma_{v(1)}$ be the measures generated by

$$
\sigma(\tau)=0
$$

for $\tau \in 2^{<\omega}$. Then the derivative $\Delta \sigma(x)$ is the set of all finite strings of $0 \mathrm{~s}$, and the measure $\sigma$ is depth zero.

For larger ordinals $\alpha$, the measures $\sigma_{u(\alpha+1)}$ and $\sigma_{v(\alpha+1)}$ do not coincide.

Lemma 3.3. For each nonzero countable ordinal $\alpha$, there are two distinct depth zero measures with range $\alpha+1=\{0,1, \ldots, \alpha\}$.

Proof. We show the existence of the depth zero measures $\sigma_{u(\alpha+1)}$ and $\sigma_{v(\alpha+1)}$ by induction on $\alpha$. As preparation, we fix, for each countable limit ordinal $\alpha$, a bijection $f_{\alpha}: \omega \rightarrow \alpha$.

For nonzero successor ordinals $\alpha$, let $\sigma_{u(\alpha+1)}$ be the measure generated by

$$
\sigma_{u(\alpha+1)}(\tau)= \begin{cases}\alpha & \text { if } \tau \text { is an almost repeater string, } \\ \sigma_{u(\alpha)}\left(\tau_{3}\right) & \text { if } \tau=\tau_{1} \frown \tau_{2} \frown \tau_{3} \text { for some repeater string } \tau_{1} \\ & \text { and xor string } \tau_{2},\end{cases}
$$

for $\tau \in 2^{<\omega}$, and let $\sigma_{v(\alpha+1)}$ be the measure generated by

$$
\sigma_{v(\alpha+1)}(\tau)= \begin{cases}\alpha & \text { if } \tau=1^{k} \text { for some integer } k, \\ \sigma_{u(\alpha)}\left(\tau^{\prime}\right) & \text { if } \tau=1^{k} \frown 0^{\frown} \tau^{\prime} \text { for some integer } k \text { and string } \tau^{\prime},\end{cases}
$$

for $\tau \in 2^{<\omega}$. For the reader's benefit, we include a figure illustrating $T_{u(2)}$ (see Figure 1)

The following observations imply that both $\sigma_{u(\alpha+1)}$ and $\sigma_{v(\alpha+1)}$ are depth zero. $u=\beta$ : If $\sigma_{u(\alpha+1)}(x)=\beta<\alpha$, then $\Delta \sigma_{u(\alpha+1)}(x)$ is the set of all finite strings of ordinals in $\beta+1$ with at least one occurrence of $\beta$.

$u=\alpha$ : If $\sigma_{u(\alpha+1)}(x)=\alpha$, then $\Delta \sigma_{u(\alpha+1)}(x)$ is the set of all finite strings of ordinals in $\alpha+1$ with at least one occurrence of $\alpha$.

$v=\beta$ : If $\sigma_{v(\alpha+1)}(x)=\beta<\alpha$, then $\Delta \sigma_{v(\alpha+1)}(x)$ is the set of all finite strings of ordinals in $\beta+1$ with at least one occurrence of $\beta$.

$v=\alpha$ : If $\sigma_{v(\alpha+1)}(x)=\alpha$, then $\Delta \sigma_{v(\alpha+1)}(x)$ is the set of all finite strings of ordinals in $\alpha+1$ containing exactly one occurrence of $\alpha$. 


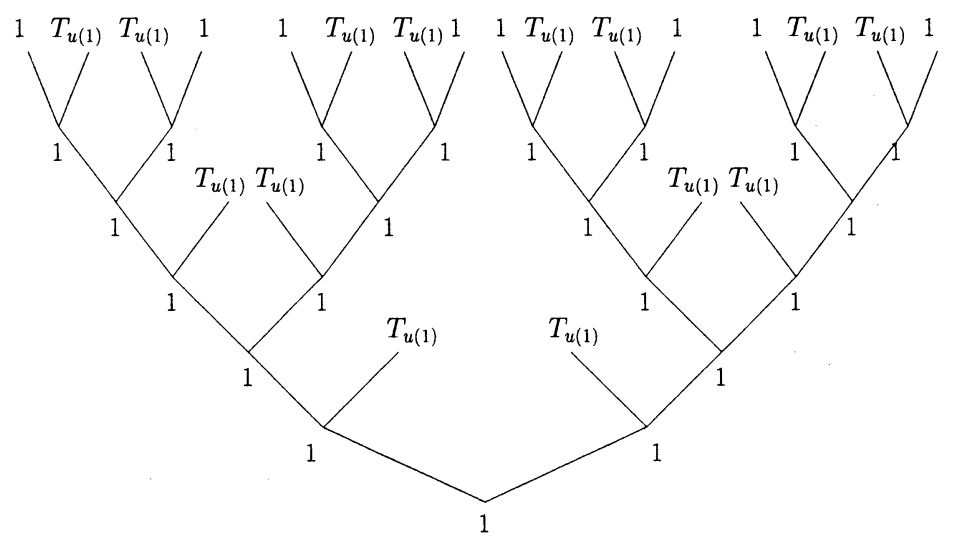

FIGURE 1. Tree for $T_{u(2)}$

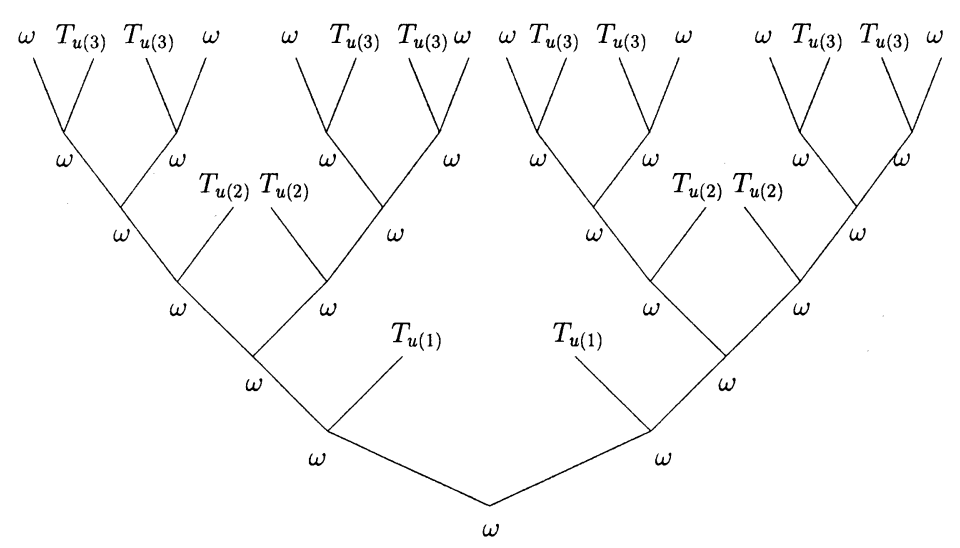

FiguRE 2. Tree for $T_{u(\omega+1)}$

Recalling the fixed bijective functions $f_{\alpha}: \omega \rightarrow \alpha$ for limit ordinals $\alpha$, let $\sigma_{u(\alpha+1)}$ be the measure generated by

$\sigma_{u(\alpha+1)}(\tau)= \begin{cases}\alpha & \text { if } \tau \text { is an almost repeater string, } \\ \sigma_{u\left(f_{\alpha}(k)\right)}\left(\tau_{3}\right) & \text { if } \tau=\tau_{1} \frown \tau_{2} \frown \tau_{3} \text { for some repeater string } \tau_{1} \text { of } \\ & \text { length } k=\frac{\left|\tau_{1}\right|-2}{2} \text { and xor string } \tau_{2},\end{cases}$

for $\tau \in 2^{<\omega}$, and let $\sigma_{v(\alpha+1)}$ be the measure generated by

$\sigma_{v(\alpha+1)}(\tau)= \begin{cases}\alpha & \text { if } \tau=1^{k} \text { for some integer } k, \\ \sigma_{u\left(f_{\alpha}(k)\right)}\left(\tau^{\prime}\right) & \text { if } \tau=1^{k} \frown 0^{\frown} \tau^{\prime} \text { for the integer } k \text { and string } \tau^{\prime},\end{cases}$

for $\tau \in 2^{<\omega}$.

Again for the reader's benefit, we include a figure illustrating $T_{u(\omega)}$ for the case when the bijection $f_{\omega}: \omega \rightarrow \omega$ is given by $n \mapsto n$ (see Figure 2).

The following observations imply that $\sigma_{u(\alpha+1)}$ and $\sigma_{v(\alpha+1)}$ are depth zero. 
$u=\beta$ : If $\sigma_{u(\alpha+1)}(x)=\beta<\alpha$, then $\Delta \sigma_{u(\alpha+1)}(x)$ is the set of all finite strings of ordinals in $\beta+1$ with at least one occurrence of $\beta$.

$u=\alpha$ : If $\sigma_{u(\alpha+1)}(x)=\alpha$, then $\Delta \sigma_{u(\alpha+1)}(x)$ is the set of all finite strings of ordinals in $\alpha+1$ with at least one occurrence of $\alpha$.

$v=\beta$ : If $\sigma_{v(\alpha+1)}(x)=\beta<\alpha$, then $\Delta \sigma_{v(\alpha+1)}(x)$ is the set of all finite strings of ordinals in $\beta+1$ with at least one occurrence of $\beta$.

$v=\alpha$ : If $\sigma_{v(\alpha+1)}(x)=\alpha$, then $\Delta \sigma_{v(\alpha+1)}(x)$ is the set of all finite strings of ordinals in $\alpha+1$ containing exactly one occurrence of $\alpha$.

We conclude that for each nonzero countable ordinal $\alpha$, there are two depth zero measures with range $\alpha+1=\{0,1, \ldots, \alpha\}$.

The transition from successor ordinals to arbitrary subsets of $\omega_{1}$ with greatest element requires the observation that the order type of any subset of $\omega_{1}$ with greatest element is a countable successor ordinal.

Proposition 3.4. For each set $S \subseteq \omega_{1}$ having exactly one element, there is a depth zero measure with range $S$. For each set $S \subseteq \omega_{1}$ with greatest element, and having more than one element, there are two distinct depth zero measures with range $S$.

Proof. Let $\alpha+1$ be the order type of $S$, noting that the order type of $S$ is a successor ordinal as $S$ is a set with a greatest element. Let $g: \alpha+1 \rightarrow S$ be the order-preserving bijection that witnesses $S$ has order type $\alpha+1$.

Let $\sigma_{u(S)}$ be the measure generated by

$$
\sigma_{u(S)}(\tau)=g\left(\sigma_{u(\alpha+1)}(\tau)\right)
$$

for $\tau \in 2^{<\omega}$, and let $\sigma_{v(S)}$ be the measure generated by

$$
\sigma_{v(S)}(\tau)=g\left(\sigma_{v(\alpha+1)}(\tau)\right)
$$

for $\tau \in 2^{<\omega}$.

The measures $\sigma_{u(S)}$ and $\sigma_{v(S)}$ are depth zero since $\sigma_{u(\alpha+1)}$ and $\sigma_{v(\alpha+1)}$ are depth zero. If $S$ has exactly one element, then $\sigma_{u(S)}$ and $\sigma_{v(S)}$ coincide as $\sigma_{u(1)}=\sigma_{v(1)}$; if $S$ has more than one element, then $\sigma_{u(S)}$ and $\sigma_{v(S)}$ are distinct as $\sigma_{u(\alpha+1)} \neq$ $\sigma_{v(\alpha+1)}$ if $\alpha>0$.

3.2. Uniqueness. Having demonstrated the existence of two depth zero measures with a specified range, we show there are at most two depth zero measures. In order to do so, we use the work of Heindorf. Specifically, we establish that every depth zero measure is pseudo-indecomposable and primitive and appeal to a result about their derivatives (see [8]).

For a measure $\sigma$ and an element $x \in \mathcal{F}$, we use the notation $\sigma_{x}$ to denote the measure given by $\sigma_{x}(z)=\sigma(x z)$ for $z \in \mathcal{F}$.

Definition 3.5. A measure $\sigma$ is pseudo-indecomposable if for every $x \in \mathcal{F}$, either $\sigma \cong \sigma_{x}$ or $\sigma \cong \sigma_{-x}$. An element $x \in \mathcal{F}$ is pseudo-indecomposable (with respect to $\sigma$ ) if $\sigma_{x}$ is pseudo-indecomposable.

Definition 3.6. A measure $\sigma$ is primitive if every element $x \in \mathcal{F}$ is a disjoint union of finitely many pseudo-indecomposable elements (with respect to $\sigma$ ).

After showing that every depth zero Boolean algebra is pseudo-indecomposable and primitive, we apply the following lemma of Heindorf's to conclude that there are no other depth zero Boolean algebras besides those described in Proposition 3.4. This is restated using our notation and language. 
Lemma 3.7 ([8]). If $\sigma$ is a pseudo-indecomposable and primitive measure with range $S$, then there are at most two possibilities for $\Delta \sigma\left(1_{\mathcal{F}}\right)$. Moreover these possibilities are not distinct in the degenerate case when $S$ has exactly one element.

Theorem 3.8. For each set $S \subseteq \omega_{1}$ having exactly one element, there is exactly one depth zero measure with range $S$, namely $\sigma_{u(S)}=\sigma_{v(S)}$. For each set $S \subseteq \omega_{1}$ with greatest element, and having more than one element, there are exactly two depth zero measures with range $S$, namely $\sigma_{u(S)}$ and $\sigma_{v(S)}$.

Proof. By Proposition 3.4 and Lemma 3.7, it suffices to show that every depth zero measure $\sigma$ is pseudo-indecomposable and primitive. We begin by showing that $\sigma_{x}$ is pseudo-indecomposable for an arbitrary element $x \in \mathcal{F}$. In order to do so, let $z \in \mathcal{F}$ be arbitrary.

As the depth of $\sigma$ was zero, the depth of $\sigma_{x}$ is also zero. Thus if $\sigma_{x}(z)=$ $\sigma_{x}\left(1_{\mathcal{F}}\right)$, then $\sigma_{x} \cong\left(\sigma_{x}\right)_{z}$ follows immediately from $\sigma_{x}$ being depth zero. If instead $\sigma_{x}(z) \neq \sigma_{x}\left(1_{\mathcal{F}}\right)$, then $\sigma_{x}(-z)=\sigma_{x}\left(1_{\mathcal{F}}\right)$ as $\sigma_{x}\left(1_{\mathcal{F}}\right)=\max \left\{\sigma_{x}(z), \sigma_{x}(-z)\right\}$, and so $\sigma_{x} \cong\left(\sigma_{x}\right)_{-z}$ follows from $\sigma_{x}$ being depth zero. Hence $\sigma_{x}$ is pseudo-indecomposable as either $\sigma_{x} \cong\left(\sigma_{x}\right)_{z}$ or $\sigma_{x} \cong\left(\sigma_{x}\right)_{-z}$ for every element $z \in \mathcal{F}$.

As $\sigma_{x}$ is pseudo-indecomposable for all $x \in \mathcal{F}$, the measure $\sigma$ is primitive. The pseudo-indecomposability of $\sigma$ is precisely the pseudo-indecomposability of $\sigma_{x}$ when $x=1_{\mathcal{F}}$.

Rather than appealing to results about pseudo-indecomposable and primitive measures, the existence of at most two depth zero measures with range $S$ can be shown directly from the depth zero hypothesis. As Theorem 3.8 implies Proposition 3.9, we keep the proof to a sketch.

Proposition 3.9. For each set $S \subseteq \omega_{1}$ with greatest element, there are at most two depth zero measures with range $S$.

Proof (Sketch). Let $\alpha=\max (S)$, which we note must exist since $S$ has a greatest element. We consider a depth zero measure $\sigma$ with range $S$. If $\beta<\alpha$ is in $S$, then there is an element $y \in \mathcal{F}$ with $\sigma(y)=\beta$. Then as $\sigma\left(1_{\mathcal{F}}\right)=\alpha=\sigma\left(1_{\mathcal{F}}-y\right)$ and $(\alpha, \beta)=\left(\sigma\left(1_{\mathcal{F}}-y\right), \sigma(y)\right) \in \Delta \sigma\left(1_{\mathcal{F}}\right)$, from the depth zero hypothesis we have that $(\alpha, \beta) \in \Delta \sigma\left(1_{\mathcal{F}}-y\right)$. Repeating this argument using $1_{\mathcal{F}}-y$ and any ordinal $\beta^{\prime}<\alpha$ in $S$, we conclude that $\left(\alpha, \beta_{1}, \ldots, \beta_{n}\right) \in \Delta \sigma\left(1_{\mathcal{F}}\right)$ for any ordinals $\beta_{1}, \ldots, \beta_{n}<\alpha$ in $S$. Moreover, no ordinal $\delta$ not in $S$ can appear in an element of $\Delta \sigma\left(1_{\mathcal{F}}\right)$.

If $(\alpha, \alpha) \in \Delta \sigma\left(1_{\mathcal{F}}\right)$, then there is a partition $1_{\mathcal{F}}=x_{1} \oplus x_{2}$ with $\sigma\left(x_{1}\right)=\alpha=$ $\sigma\left(x_{2}\right)$. As $\sigma$ was depth zero and $\sigma\left(x_{1}\right)=\sigma\left(1_{\mathcal{F}}\right)=\sigma\left(x_{2}\right)$, we must have $(\alpha, \alpha) \in$ $\Delta \sigma\left(x_{1}\right), \Delta \sigma\left(x_{2}\right)$. Then there exist partitions $x_{1}=x_{11} \oplus x_{12}$ and $x_{2}=x_{21} \oplus x_{22}$ with $\sigma\left(x_{11}\right)=\alpha=\sigma\left(x_{12}\right)$ and $\sigma\left(x_{21}\right)=\alpha=\sigma\left(x_{22}\right)$. Repeating this argument, it follows that $(\alpha, \ldots, \alpha) \in \Delta \sigma\left(1_{\mathcal{F}}\right)$.

It follows that if $(\alpha, \alpha) \notin \Delta \sigma\left(1_{\mathcal{F}}\right)$, then $\Delta \sigma\left(1_{\mathcal{F}}\right)$ consists of the set of all finite sequences of ordinals from $S$ containing exactly one occurrence of $\alpha$. Otherwise $\Delta \sigma\left(1_{\mathcal{F}}\right)$ consists of the set of all finite sequences of ordinals from $S$ containing at least one occurrence of $\alpha$.

\section{Computable Rank $\omega$, Depth zero Boolean algebras}

Having characterized the algebraic structure of the depth zero Boolean algebras in Section 3, we turn our attention to characterizing those which have effective presentations. Since there is an effective transition between a measure and the 
corresponding Boolean algebra, it may seem reasonable to believe that a Boolean algebra has a computable presentation if and only if it has a computable measure. However this is far from true, as the following theorem demonstrates.

Theorem 4.1. Let $S \subseteq \omega+1$ be a set with greatest element. Then the following are equivalent:

(1) The Boolean algebra $\mathcal{B}_{u(S)}$ is computable.

(2) The Boolean algebra $\mathcal{B}_{v(S)}$ is computable.

(3) The set $S \backslash\{\omega\}$ is $\Sigma_{(2 n+3)}^{0}$.

The proof of Theorem 4.1 is lengthy and contains disparate techniques, and thus it is split into Section 4.1 and Section 4.2 where we show (1), (2) $\Longrightarrow$ (3) and $(3) \Longrightarrow(1),(2)$, respectively.

4.1. Proof of Theorem 4.1, (1), (2) $\Longrightarrow$ (3). In order to show that $S \backslash\{\omega\}$ is $\Sigma_{(2 n+3)}^{0}$ if $\mathcal{B}_{u(S)}$ or $\mathcal{B}_{v(S)}$ is computable, we give, uniformly for each $n \in \omega$, a computable infinitary sentence $\varphi_{n}$ of complexity $\Sigma_{2 n+3}^{0}$. The sentence $\varphi_{n}$ will be constructed so that the depth zero Boolean algebras $\mathcal{B}_{u(S)}$ and $\mathcal{B}_{v(S)}$ satisfy $\varphi_{n}$ if and only if $n \in S$.

We start by noting the complexity of various well-known arithmetical formulas (see [2, for example). There is a finitary $\Pi_{2}^{0}$ formula atomless $(x)$ saying that $x$ is atomless. Also, for each $n$, there is a computable infinitary $\Pi_{2 n+1}^{0}$ formula atom ${ }_{n}(x)$ saying that $x$ is an $n$-atom.

Using these, we define the sentences $\varphi_{n}$ and argue that the depth zero Boolean algebras $\mathcal{B}_{u(S)}$ and $\mathcal{B}_{v(S)}$ satisfy $\varphi_{n}$ if and only if $n \in S$.

Definition 4.2. Let $\varphi_{0}$ be the finitary sentence

$$
\varphi_{0}:=(\exists x)[\operatorname{atomless}(x)] .
$$

For $n>0$, let $\varphi_{n}$ be the computable infinitary sentence

$$
\begin{aligned}
\varphi_{n}:= & (\exists x)\left[(\forall y \leq x)\left[\neg \operatorname{atom}_{n}(y)\right] \&\right. \\
& \left.\bigwedge_{k \in \omega}\left(\exists x_{1}, \ldots, x_{k}\right)(\forall i \neq j)\left[x_{i}<x \& \operatorname{atom}_{n-1}\left(x_{i}\right) \& x_{i} x_{j}=0\right]\right]
\end{aligned}
$$

Proposition 4.3. The depth zero Boolean algebras $\mathcal{B}_{u(S)}$ and $\mathcal{B}_{v(S)}$ satisfy $\varphi_{n}$ if and only if $n \in S$.

Proof. We consider the case when $n=0$ separately from the case when $n>0$. If $n=0$, then $\mathcal{B}_{u(S)}$ and $\mathcal{B}_{v(S)}$ satisfy $\varphi_{0}$ if and only if they have an atomless element, which happens if and only if $0 \in S$.

If $n>0$, then $\mathcal{B}_{u(S)}$ and $\mathcal{B}_{v(S)}$ satisfy $\varphi_{n}$ if and only if they have an element bounding infinitely many $(n-1)$-atoms but no $n$-atoms. An element witnessing this must be part of the perfect kernel not bounding any $n$-atoms. For if it were not part of the perfect kernel, then it would be superatomic, an impossibility. The measure of any such element is thus $n$, which happens if and only if $n \in S$.

We finish by showing that (1), (2) $\Longrightarrow(3)$.

Theorem 4.4. If $\mathcal{B}_{u(S)}$ is computable, then $S \backslash\{\omega\}$ is $\Sigma_{(2 n+3)}^{0}$. If $\mathcal{B}_{v(S)}$ is computable, then $S \backslash\{\omega\}$ is $\Sigma_{(2 n+3)}^{0}$. 
Proof. We begin by analyzing the quantifier complexity of the $\varphi_{n}$. Since atomless $(x)$ has a computable $\Pi_{2}^{0}$ representation, the sentence $\varphi_{0}$ is computable $\Sigma_{3}^{0}$. Since $\operatorname{atom}_{n}(x)$ has a computable $\Pi_{2 n+1}^{0}$ representation, the sentence $\varphi_{n}$ is computable $\Sigma_{2 n+3}^{0}$ for $n>0$.

As a consequence of Proposition 4.3 it follows that if $\mathcal{B}_{u(S)}$ (respectively $\mathcal{B}_{v(S)}$ ) is computable, then $S$ is $\Sigma_{(2 n+3)}^{0}$.

4.2. Proof of Theorem 4.1, (3) $\Longrightarrow(1),(2)$. In order to show that $\mathcal{B}_{u(S)}$ and $\mathcal{B}_{v(S)}$ are computable if $S \backslash\{\omega\}$ is $\Sigma_{(2 n+3)}^{0}$, we construct computable copies of them from an index $e$ witnessing that $S \backslash\{\omega\}$ is $\Sigma_{(2 n+3)}^{0}$. Before doing so, we prove a lemma which will be iterated in the construction of $\mathcal{B}_{u(S)}$ and $\mathcal{B}_{v(S)}$. Lemma4.6 is a modification of the following theorem of Ash (see [1]), which is itself a modification of a theorem of Watnick (see [19]).

Theorem 4.5 ([1]). There is a procedure, uniform in $\alpha$ and in a $\Delta_{2 \alpha+1}^{0}$ index for the atomic diagram $D(\mathcal{A})$ of a linear order $\mathcal{A}$ with distinguished least element, which yields a $\Delta_{1}^{0}$ linear order $\mathcal{L}$ such that $\mathcal{L} \cong \omega^{\alpha} \cdot \mathcal{A}$.

For depth zero Boolean algebras with $S \subseteq \omega+1$, it suffices to consider only finite ordinals $\alpha$. Thurber's sketch of Theorem 4.5 (see [17]) therefore serves as an outline to prove the main technical lemma needed. We use $\eta$ to denote the order type of the rational numbers and Fin to denote any order type consisting of at least two, but at most finitely many, points.

Lemma 4.6. There is a procedure, uniform in a $\Delta_{3}^{0}$ index for the atomic diagram $D(\mathcal{A})$ of a linear order $\mathcal{A}=(A: \prec)=\left(\left\{a_{0}, a_{1}, \ldots\right\}: \prec\right)$ with distinguished least element $a_{0}$ and an index for a $\Sigma_{3}^{0}$ predicate $\exists n \forall u \exists v R(n, u, v)$, which yields a $\Delta_{1}^{0}$ linear order $\mathcal{L}$ such that $\mathcal{L} \cong \sum_{a \in A} \mathcal{L}_{a}$, where $\mathcal{L}_{a_{n}} \cong 1+\eta+\omega$ if $\forall u \exists v R(n, u, v)$ and $\mathcal{L}_{a_{n}} \cong \omega$ otherwise.

Proof. By hypothesis, we have a $\Delta_{3}^{0}$ function $h$ such that $h(n)$ codes the atomic diagram of $\mathcal{A}$ restricted to $\left\{a_{0}, a_{1}, a_{2}, \ldots, a_{n}\right\}$. From the Limit Lemma, we obtain a $\Delta_{2}^{0}$ function $g(n, s)$ and a $\Delta_{1}^{0}$ function $f(n, s, k)$ (uniformly from an index for $h$ ) such that

$$
\lim _{s} g(n, s)=h(n) \quad \text { and } \quad \lim _{k} f(n, s, k)=g(n, s) .
$$

We impose the following constraints on the approximations $g(n, s)$ and $f(n, s, k)$ without any loss of uniformity.

(1) The approximations $g(n, s)$ say that $a_{0}$ is the least element of $\mathcal{A}$ for all $n$ and $s$.

(2) The approximations $f(n, s, k)$ say that $a_{0}$ is the least element of $\mathcal{A}$ for all $n, s$, and $k$.

(3) The approximations $g(n, s)$ satisfy $g(m, s) \subset g(n, s)$ for all $s$ and $m<n$; i.e., the linear order specified by $g(n, s)$ extends the linear order specified by $g(m, s)$.

(4) The approximations $f(n, s, k)$ satisfy $f(m, s, k) \subset f(n, s, k)$ for all $s, k$, and $m<n$; i.e., the linear order specified by $f(n, s, k)$ extends the linear order specified by $f(m, s, k)$.

Using $f$ we will try to build, for each point $a_{n}$ in $\mathcal{A}$, a linear order $\mathcal{L}_{n}$ at the location in $\mathcal{L}$ where we believe $a_{n}$ to be. The linear order $\mathcal{L}_{n}$ (termed a block) will 
consist of three parts (termed segments): a singleton segment, a dynamic segment to the right of the singleton segment, and a discrete segment to the right of the dynamic segment.

The singleton segment will start as a single point and never have additional points added to it.

The dynamic segment will start as a single point and, in the limit, have order type $\eta$ or FIN. Whenever a new witness is found for $a_{n}$ for the $\Sigma_{3}^{0}$ predicate, i.e., a $v$ is discovered such that $R(n, u, v)$ for the least $u$ with no such pre-existing $v$, the dynamic segment is densified. More specifically, new elements are added at either end of the dynamic segment and between every pair of already existing elements in the dynamic segment. In order to help track for which $u$ witnesses $v$ have been found for $n$, parameters $u_{n}$ and $v_{n}$ are used.

The discrete segment will also start as a single point but, in the limit, have order type $\omega$. At each stage, a new element is added to the right end of the discrete segment. The discrete segment will also serve as a garbage collection for unwanted blocks that were mistakenly created as a consequence of misapproximations of $h(n)$ by $g(n, s)$ or $f(n, s, k)$.

At a given stage $k$, we will act on behalf of a pair $(n, s)=\left(n_{k}, s_{k}\right)$ for some $n \leq s \leq k$ using the approximation $f(n, s, k)$. Several possibilities exist, which we describe informally. Our approximation $f(n, s, k)$ may suggest new work, in which case we begin a new block $\mathcal{L}_{n}$. Our approximation $f(n, s, k)$ may agree with previous work, in which case we simply expand all linear orders $\mathcal{L}_{m}$ for $m \leq n$. Our approximation $f(n, s, k)$ may disagree with the previous guess about the location of $a_{n}$, in which case we attach each $\mathcal{L}_{m}$ for every $m \geq n$ to its predecessor by associating its points with its predecessor's discrete segment. Depending on certain conditions, elaborated upon later, we begin building a new instantiation of $\mathcal{L}_{n}$ at the new location of $a_{n}$ or detach a previously attached instantiation of $\mathcal{L}_{n}$.

Since we may build a block $\mathcal{L}_{n}$ for $a_{n}$ at a wrong location relative to $\mathcal{L}_{m}$ for $m<n$ because of a misapproximation, multiple instantiations of $\mathcal{L}_{n}$ for $a_{n}$ may be started. Ones believed to be incorrectly placed will be attached to their predecessor as suggested above. In order to help determine whether a previously attached version should be detached, each instantiation of a block $\mathcal{L}_{n}$ is tagged with a tuple $(\tilde{n}, \tilde{s}, \tilde{k})$. The value of $\tilde{s}$ will be the block's approximation priority, which will be the value of $s_{k}$ when the block is begun. The value of $\tilde{k}$ will be the block's chronological priority, which will be the value of $k$ when the block is begun. If the chronological priorities of blocks $\mathcal{L}_{n}$ and $\mathcal{L}_{n^{\prime}}$ are $\tilde{k}$ and $\tilde{k}^{\prime}$, we say that $\mathcal{L}_{n}$ is chronologically older than $\mathcal{L}_{n^{\prime}}$ if $\tilde{k}<\tilde{k}^{\prime}$.

In order to help track the construction and aid its success, an auxiliary function $r(n, s, k)$ and parameters $n_{k}$ and $s_{k}$ are used. The partial function $r(n, s, k)$ describes the approximation when we last acted on behalf of the pair $(n, s)$. More specifically, the value of $r(n, s, k)$ is $f\left(n, s, k^{\prime}\right)$, where $k^{\prime}$ is the last stage when we acted on behalf of $(n, s)$. The parameters $n_{k}$ and $s_{k}$ specify the pair $(n, s)=\left(n_{k}, s_{k}\right)$ acted on behalf of at stage $k$.

Construction: The construction involves $\omega$ many stages and builds a linear order $\mathcal{L}$ with universe $\left\{b_{0}, b_{1}, b_{2}, \ldots\right\}$. At stage 0 , we fix $b_{0}$ as the least element of $\mathcal{L}$, tag the block with the tuple $(\tilde{n}, \tilde{s}, \tilde{k})=(0,0,0)$, and commit ourselves to never putting anything before it. We also set the parameters $n_{0}$ and $s_{0}$ to 0 and put $r(0,0,0)=f(0,0,0)$. 
At the end of each stage $k-1$ for $k \geq 1$, we assume that various blocks have been started and tagged with tuples $(\tilde{n}, \tilde{s}, \tilde{k})$ with $\tilde{n} \leq \tilde{s} \leq \tilde{k}$, that at stage $k-1$ we acted on behalf of the pair $\left(n_{k-1}, s_{k-1}\right)$, and that we have defined a partial function $r(n, s, k-1)$ for $n \leq s \leq k-1$ which describes our last action for the pair $(n, s)$.

Each stage $k>0$ proceeds in three substages: defining the values of the parameters $n_{k}$ and $s_{k}$, acting on behalf of the pair $\left(n_{k}, s_{k}\right)$, and enlarging all active blocks.

Substage 0 defines the values of the parameters $n_{k}$ and $s_{k}$ in the following manner. Let $s_{k}$ be the least $s \leq s_{k-1}$ such that $f(n, s, k) \neq r(n, s, k-1)$ for some $n \leq s$ if such an $s$ exists; otherwise let $s_{k}=s_{k-1}+1$. Then let $n_{k}$ be the least $n \leq s_{k}$ such that $f\left(n, s_{k}, k\right) \neq r\left(n, s_{k}, k-1\right)$ if such an $n$ exists; otherwise let $n_{k}=s_{k}$.

Substage 1 acts on behalf of the pair $\left(n_{k}, s_{k}\right)$ and defines more of the function $r(n, s, k)$ in the following manner. Our action depends on which of the following four scenarios occurs: we haven't yet begun building a block $L_{n_{k}}$; we haven't yet acted for $\left(n_{k}, s_{k}\right)$, but what we've done so far for $\left(n_{k}, s\right)$ for $s<s_{k}$ seems correct; we agree with what we've done so far for $\left(n_{k}, s_{k}\right)$; we think the block built for $\left(n_{k}, s_{k}\right)$ is at the wrong place.

(1) Scenario: We haven't yet begun building a block $\mathcal{L}_{n_{k}}$. More precisely, the function $r(n, s, k)$ is undefined at $n_{k}$ for all values of $s$ and $k$.

Action: We start a new block $\mathcal{L}_{n_{k}}$ for $a_{n_{k}}$, $\operatorname{tag} \mathcal{L}_{n_{k}}$ with $\left(n_{k}, s_{k}, k\right)$, initialize the parameters $u_{n_{k}}$ and $v_{n_{k}}$, and update the function $r(n, s, k)$ appropriately. More precisely:

We begin the block $\mathcal{L}_{n_{k}}$ in the place indicated by $f\left(n_{k}, s_{k}, k\right)$ relative to the blocks $\mathcal{L}_{m}$ for $m<n_{k}$. In particular, we insert a new element as the singleton segment of $\mathcal{L}_{n_{k}}$, a new element as the dynamic segment of $\mathcal{L}_{n_{k}}$, and a new element as the discrete segment of $\mathcal{L}_{n_{k}}$.

We tag the block $\mathcal{L}_{n_{k}}$ with the tuple $(\tilde{n}, \tilde{s}, \tilde{k})=\left(n_{k}, s_{k}, k\right)$, thus giving it approximation priority $s_{k}$ and chronological priority $k$. These will never change. We initialize the parameters $u_{n_{k}}$ and $v_{n_{k}}$ (which are specific to this instantiation of $\left.\mathcal{L}_{n_{k}}\right)$ to zero. We also set $r\left(n_{k}, s_{k}, k\right)=f\left(n_{k}, s_{k}, k\right)$ and set $r(n, s, k)=r(n, s, k-1)$ for all $n<n_{k}$ and $s \leq s_{k}$.

(2) Scenario: We haven't yet acted for $\left(n_{k}, s_{k}\right)$, but what we've done so far for $\left(n_{k}, s\right)$ for $s<s_{k}$ seems correct. More precisely, the function $r(n, s, k)$ is undefined at $n_{k}$ and $s_{k}$ for all values of $k$, but $f\left(n_{k}, s_{k}, k\right)=r\left(n_{k}, s^{\prime}, k-1\right)$ for all $s^{\prime}$ with $\tilde{s} \leq s^{\prime}<s_{k}$, where $\tilde{s}$ is the approximation priority of the active instantiation of the block $\mathcal{L}_{n_{k}}$.

Action: We update the function $r(n, s, k)$ appropriately. More precisely:

We set $r\left(n_{k}, s_{k}, k\right)=f\left(n_{k}, s_{k}, k\right)$ and set $r(n, s, k)=r(n, s, k-1)$ for all $n \leq n_{k}$ and $s \leq s_{k}$ (excepting $n=n_{k}$ and $s=s_{k}$ ).

(3) Scenario: We agree with what we've done so far for $\left(n_{k}, s_{k}\right)$. More precisely, $f\left(n_{k}, s_{k}, k\right)=r\left(n_{k}, s_{k}, k-1\right)$.

Action: We update the function $r(n, s, k)$ appropriately. More precisely:

We set $r(n, s, k)=r(n, s, k-1)$ for all $n \leq n_{k}$ and $s \leq s_{k}$.

(4) Scenario: We think the block built for $\left(n_{k}, s_{k}\right)$ is at the wrong place. More precisely, $f\left(n_{k}, s_{k}, k\right) \neq r\left(n_{k}, s_{k}, k-1\right)$.

Action: We attempt to correct our previous "mistake" by attaching the blocks thought wrongly built to their predecessor. We then build $\mathcal{L}_{n_{k}}$ at the new location, either by starting a new instantiation of $\mathcal{L}_{n_{k}}$ or detaching 
a previously attached instantiation. We then update the function $r(n, s, k)$ appropriately. More precisely:

Each block $\mathcal{L}_{m}$ with $m \geq n_{k}$ is attached to the block $\mathcal{L}_{\ell(m)}$ immediately to its left, beginning with $\mathcal{L}_{n_{k}}$ and counting upwards. We note the block $\mathcal{L}_{\ell(m)}$ must exist for each $m$ as a result of Constraint 2. The attachment is done by associating all the points in $\mathcal{L}_{m}$ with the discrete segment of the linear order $\mathcal{L}_{\ell(m)}$. The points from $\mathcal{L}_{m}$ retain the tuple with which they were tagged at their creation.

We illustrate an attachment involving several blocks (see Figure 3).

The instantiation of the block $\mathcal{L}_{n_{k}}$ at the location given by $f\left(n_{k}, s_{k}, k\right)$ is either built from scratch or possibly detached from the block $\mathcal{L}_{m}$ immediately to the left of this location. The block is detached only if:

(a) It was previously started at some stage $k^{\prime}$ at this location relative to $a_{\ell}$ for $\ell<n_{k}$, tagged with a tuple $(\tilde{n}, \tilde{s}, \tilde{k})$ satisfying $\tilde{n}=n_{k}$ and $\tilde{s} \leq s_{k}$, and attached to $\mathcal{L}_{m}$ at some stage $k^{\prime \prime}$ with $k^{\prime}<k^{\prime \prime}<k$.

(b) There is no $t$ with $\tilde{s}<t<s_{k}$ such that $f\left(n_{k}, s_{k}, k\right) \neq f\left(n_{k}, t, k\right)$.

(c) Detaching the block would result in no chronologically older block being detached.

The detachment is done by splitting off all the elements in the block $\mathcal{L}_{m}$ that were attached to $L_{m}$ at the stage $k^{\prime \prime}$ or were added to the block $\mathcal{L}_{m}$ to the right of these elements.

We illustrate a detachment in which all the necessary conditions are assumed to have been met (see Figure 4).

If no detachment occurs, we begin a new instantiation of the block $\mathcal{L}_{n_{k}}$ at the location given by $f\left(n_{k}, s_{k}, k\right)$. In particular, we insert a new element as the singleton element segment of $\mathcal{L}_{n_{k}}$, a new element as the dynamic segment of $\mathcal{L}_{n_{k}}$, and a new element as the discrete segment of $\mathcal{L}_{n_{k}}$. We also initialize new parameters $u_{n_{k}}$ and $v_{n_{k}}$ (specific to this instantiation of $\mathcal{L}_{n_{k}}$ ) to zero.

We set $r\left(n_{k}, s_{k}, k\right)=f\left(n_{k}, s_{k}, k\right)$ and set $r(n, s, k)=r(n, s, k-1)$ for all $n \leq n_{k}$ and $s \leq s_{k}$ (except $n=n_{k}$ and $s=s_{k}$ ).

Substage 2 enlarges all blocks $\mathcal{L}_{n}$ for $n \leq n_{k}$ in the following manner. The dynamic segment is densified depending on whether $R\left(n, u_{n}, v_{n}\right)$ holds or not. If $R\left(n, u_{n}, v_{n}\right)$ holds, then $u_{n}$ is incremented, $v_{n}$ is reset to 0 , and the dynamic segment is densified by adding new elements at either end and between every pair of already existing elements of the dynamic segment. If $R\left(n, u_{n}, v_{n}\right)$ fails to hold, we leave $u_{n}$ unchanged, increment $v_{n}$, and do not modify the dynamic segment. Independent of whether $R\left(n, u_{n}, v_{n}\right)$ holds, a new element is added at the right end of the discrete segment.

This completes the description of the construction.

Verification: In order to be sure that our construction yields $\sum_{a \in A} \mathcal{L}_{a}$, we verify that a block $\mathcal{L}_{n}$ of the correct order type is built at the correct location relative to blocks $\mathcal{L}_{m}$ for $m \leq n$. As blocks will be absorbed and unabsorbed into other blocks through the processes of attachment and detachment, the owner of an instantiation of a block $\mathcal{L}_{n}$ at stage $k$ is the block $\mathcal{L}_{n^{\prime}}$ such that $\mathcal{L}_{n}$ is part of the discrete segment of $\mathcal{L}_{n^{\prime}}$ at stage $k$ (allowing the possibility of $n=n^{\prime}$ ). A stage $k$ where $h(n) \subseteq f\left(n_{k}, s_{k}, k\right)$ is said to be a true stage for $n$.

The correctness of the construction will be a consequence of the following claims. 

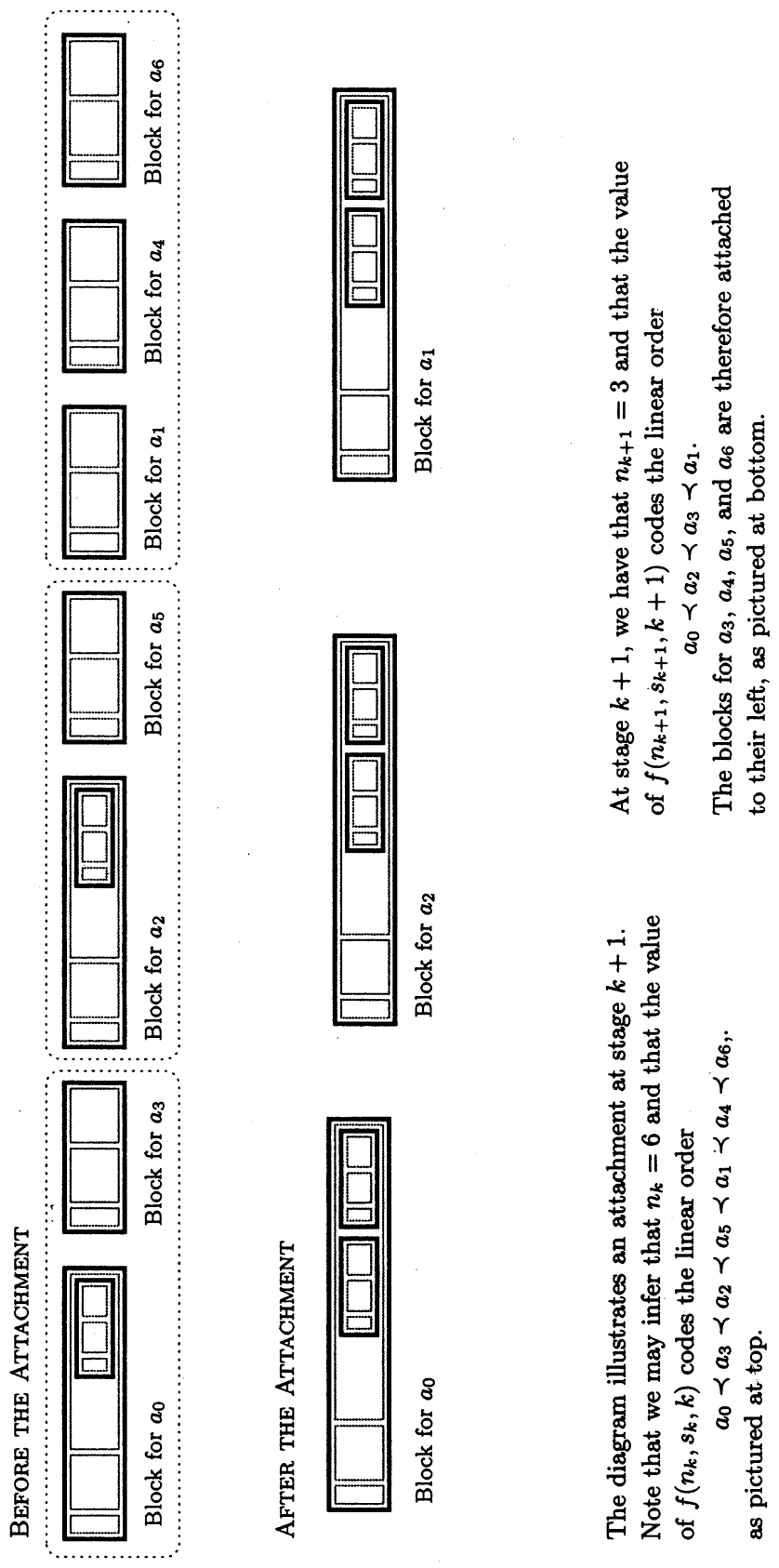

Figure 3. Block Attachment

- All wrongly started pieces are eventually found to be wrongly started and permanently attached.

- For each $n$, a block $\mathcal{L}_{n}$ is built for $a_{n}$ at the correct location relative to $a_{\ell}$ for $\ell<n$ that is detached again after each time it is attached. 

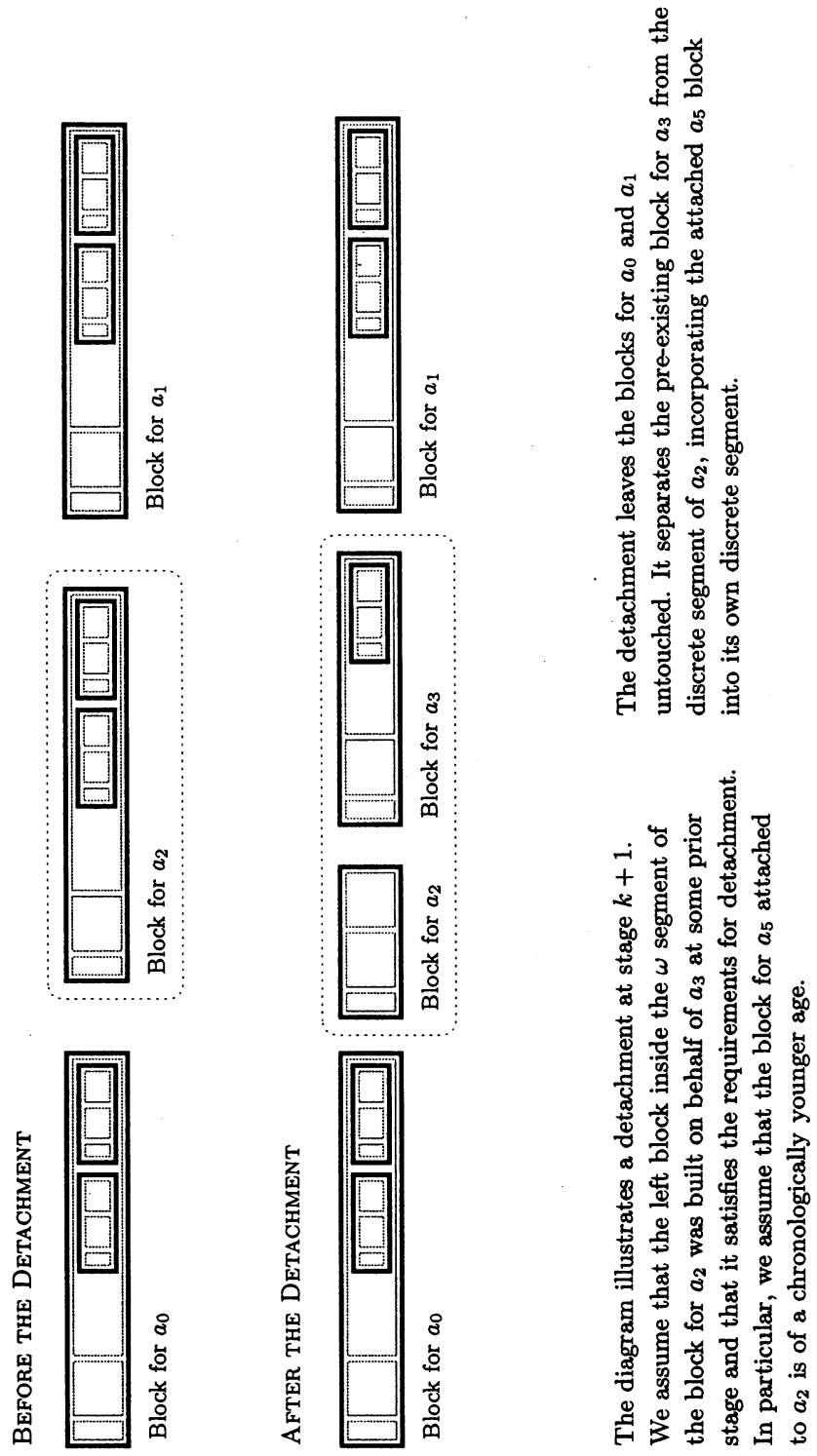

FiguRE 4. Block Detachment

- For each $n$, the block $\mathcal{L}_{n}$ built for $a_{n}$ at the correct location relative to $a_{\ell}$ for $\ell<n$ is of the correct isomorphism type.

Roughly speaking, the first claim guarantees that we have no extra elements in the linear order $\mathcal{L}$ while the second and third claims together guarantee that we have at least the appropriate elements in the linear order $\mathcal{L}$. Before formalizing and proving these claims, we show the following combinatorial claims about the construction. 
Claim 4.6.1. For a fixed $s$, there are only finitely many $k$ such that $s_{k}=s$. Thus, for a fixed $s$, there are only finitely many $k$ such that $s_{k} \leq s$.

Proof. We show there are only finitely many $k$ such that $s_{k}=s$ by induction on $s$. For $s=0$, we have $s_{k}=0$ if and only if $k=0$ as there is only one possible atomic diagram for the linear order consisting only of $a_{0}$. Consequently the value of $f(0,0, k)$ is constant in $k$, so $r(0,0, k)$ will be constant in $k$, and thus the condition $f(0,0, k) \neq r(0,0, k-1)$ will never be met.

Assuming $s_{k}=s$ for only finitely many $k$, we argue that $s_{k}=s+1$ for only finitely many $k$. If $s_{k}=s+1$, either $s_{k-1}=s$ or $f(n, s+1, k) \neq r(n, s+1, k-1)$ for some $n \leq s+1$. By the inductive hypothesis, the former can happen only finitely often. Fixing $n$, let $\hat{k}$ be least such that $f(n, s+1, k)=g(n, s+1)$ for all $k>\hat{k}$. For $k \geq \hat{k}$, we will have the inequality $f(n, s+1, k) \neq r(n, s+1, k-1)$ for at most one $k$; if we witness this inequality at stage $k$, we will set $r(n, s+1, k)=f(n, s+1, k)$ and both $f(n, s+1, \cdot)$ and $r(n, s+1, \cdot)$ will be constant and equal thereafter. Thus the latter condition can also happen only finitely often.

The first statement in the claim then follows by induction, from which the second statement in the claim immediately follows.

Claim 4.6.2. For each $n$, there are infinitely many true stages for $n$.

Proof. We begin by noting that if $k$ is a true stage for $n$, then $k$ is a true stage for all $m<n$ by Constraint 4 . Also as $n_{k} \leq k$ for all $k$, a stage $k$ can only be a true stage for finitely many $n$. We therefore need only show that for each $n$, then there is a stage $k$ such that $k$ is a true stage for $n$.

In order to do so, fix $n$ and let $\hat{s}$ be least such that $g(n, s)=h(n)$ for all $s \geq \hat{s}$. Let $\hat{k}$ be least such that $f(n, \hat{s}, k)=g(n, \hat{s})$ for all $k \geq \hat{k}$. Finally, let $s^{\prime}$ be least greater than $\hat{s}$ such that $f\left(n, s^{\prime}, \cdot\right)$ has not yet converged to $g(n, s)$ by stage $\hat{k}$. If no such $s^{\prime}$ exists, then the claim follows as any stage $k$ with $n_{k} \geq n$ and $s_{k} \geq \hat{s}$ is then a true stage for $n$.

Let $\hat{k}^{\prime}$ be least such that $f\left(n, s^{\prime}, k\right)=g\left(n, s^{\prime}\right)$ for all $k \geq \hat{k}^{\prime}$. At stage $\hat{k}^{\prime}$, we will have $n_{\hat{k}^{\prime}} \leq n$ and $s_{\hat{k}^{\prime}} \leq s^{\prime}$. If $n_{\hat{k}^{\prime}}=n$ and $s_{\hat{k}^{\prime}}=s^{\prime}$, then $\hat{k}^{\prime}$ will be a true stage for $n$. Otherwise, the first stage $k>\hat{k}^{\prime}$ with $n_{k} \geq n$ and $s_{k} \geq s^{\prime}$ will be a true stage for $n$.

We continue by formalizing and proving the three claims.

Claim 4.6.3. If a block $\mathcal{L}_{n}$ for $a_{n}$ is started at the wrong location relative to $\mathcal{L}_{m}$ for $m<n$, then there is a stage $k$ at which $\mathcal{L}_{n}$ is attached and never detached afterwards.

Proof. We prove the claim by using induction on the chronological priority of the wrongly started block $\mathcal{L}_{n}$. The oldest chronological block, the block $\mathcal{L}_{0}$ created at stage 0 , is never found to be wrongly started by Constraint 2 . Proceeding with the induction, we assume that $n>0$ and that the claim is valid for all wrongly started blocks with stronger chronological priority.

A block $\mathcal{L}_{n}$ for $a_{n}$ can be wrongly started for several reasons: if $g(n, s)$ was wrong but we guessed correctly for it, i.e., $f(n, s, k)=g(n, s) \neq h(n)$; if $g(n, s)$ was correct but we guessed incorrectly for it, i.e., $f(n, s, k) \neq g(n, s)=h(n)$; and if $g(n, s)$ was wrong and we guessed incorrectly for it, i.e., $f(n, s, k) \neq g(n, s) \neq h(n) \neq f(n, s, k)$. 
In all cases, there is an $\hat{s}$ such that $g(n, s)=h(n)$ for all $s \geq \hat{s}$ and a $\hat{k}$ such that $f(n, \hat{s}, k)=g(n, \hat{s})=h(n)$ for all $k \geq \hat{k}$. The strategy in Scenario 4 implies that we will not detach this block for the sake of a pair $(n, s)$ for any $s \geq \hat{s}$ after stage $\hat{k}$. Moreover, as $s_{k}<\hat{s}$ for only finitely many $k$ by Claim 4.6.1, this block will be detached only finitely often for $(n, s)$ for $s<\hat{s}$.

Moreover, as we are assuming the claim for all blocks of stronger chronological priority, each of these blocks will be detached wrongly only finitely often as a consequence of their own actions. Thus $\mathcal{L}_{n}$ will eventually be attached and never detached afterwards.

We note that if $f(n, s, k) \neq g(n, s) \neq h(n)=f(n, s, k)$ for some $n, s$, and $k$, then the approximation errors seem to "cancel" each other. However, at some later stage, $f(n, s, k)$ will converge to $g(n, s)$, and shortly afterward the wrongly started block will be permanently attached to its predecessor.

Claim 4.6.4. For each $n$, there is a block $\mathcal{L}_{n}$ built for $a_{n}$ at the correct location relative to $\mathcal{L}_{m}$ for $m<n$ that is eventually detached after every time it is attached.

Proof. We establish the claim ignoring the possibly detrimental effects of chronological priorities. By this we mean that every such block will be eventually detached if Detachment Condition (c) is not required. After doing so, we argue that the claim holds after considering chronological priorities.

Fixing $n$, let $\hat{s}$ be least such that $g(n, s)=h(n)$ for all $s \geq \hat{s}$. Let $\hat{k}$ be least such that $f(n, \hat{s}, k)=g(n, \hat{s})=h(n)$ for all $k \geq \hat{k}$. Consider any stage $\ell \geq \hat{k}$ such that the following criteria are met:

(1) For all stages $k \geq \ell, s_{k} \geq \hat{s}$.

(2) The stage $\ell$ is a true stage for $n$.

We argue that the block $\mathcal{L}_{n}$ existing for $a_{n}$ at stage $\ell$ is eventually detached after every time it is attached, ignoring the possibly detrimental effects of chronological priorities. We therefore assume that this block is later attached on behalf of some pair $\left(n^{\prime}, s^{\prime}\right)$ with $n^{\prime} \leq n$ and $s^{\prime}>\hat{s}$. We note that these inequalities on $n^{\prime}$ and $s^{\prime}$ must be satisfied as a result of Constraint 4 and the hypotheses on $\ell$.

As this block was built at the correct location relative to $a_{m}$ for $m<n$, the attachment must have been a result of $f\left(n^{\prime}, s^{\prime}, \cdot\right)$ misapproximating $g\left(n^{\prime}, s^{\prime}\right)$. We note that the attachment could not have been a result of $g\left(n^{\prime}, \cdot\right)$ misapproximating $h\left(n^{\prime}\right)$ as a consequence of Constraint 3 .

At some point after the attachment on behalf of the pair $\left(n^{\prime}, s^{\prime}\right)$, there will be a stage $k$ such that the pair $\left(n_{k}, s_{k}\right)$ satisfies $n_{k}=n$ and $f(n, s, k)=g(n, s)=h(n)$ for all $\hat{s} \leq s \leq s_{k}$. At this stage $k$, this block will be detached (ignoring chronological priorities) as the other conditions required for a block detachment are necessarily met. We conclude that, ignoring the possibly detrimental effects of chronological priorities, the claim holds.

We continue by showing, using induction on $n$, that the claim holds after considering the possibly detrimental effects of chronological priorities. Assuming the claim for all $m<n$, we show it is true for $n$. We may assume that the block $\mathcal{L}_{n}$ discussed will eventually have an older block permanently attached to it, else we are done with the claim. Consequently, we will create a new instantiation of the block $\mathcal{L}_{n}$ as the older block will prevent a detachment. We may assume that this instantiation exists at a stage $\ell^{\prime}$ later than $\ell$ such that all true blocks $\mathcal{L}_{m}$ for $m<n$ 
have been created and $\ell^{\prime}$ is a true stage for $n$. We note that such a stage must exist as there are infinitely many true stages for $n$ and by the inductive hypothesis.

We argue that the instantiation of the block $\mathcal{L}_{n}$ existing at stage $\ell^{\prime}$ satisfies the claim. In order to do so, we need only show that it will never have a chronologically older block attached to it. As all the true blocks $\mathcal{L}_{m}$ have been created for $m<n$ and $\ell^{\prime}$ is a true stage, the instantiations of the blocks for $m<n$ at stage $\ell^{\prime}$ must be the true instantiations. It follows that no chronologically older incorrect blocks will ever be attached to $\mathcal{L}_{n}$ (at a true stage).

By induction, we conclude that the claim holds after considering the possibly detrimental effects of chronological priorities.

Claim 4.6.5. For the block $\mathcal{L}_{n}$ at the correct location that is eventually detached after every time it is attached, the order type is $1+\eta+\omega$ if $\forall u \exists v R(n, u, v)$ and $\omega$ otherwise.

Proof. Claim 4.6.4 established that there is a block $\mathcal{L}_{n}$ at the correct location that is detached again after each time it is attached, and so this instantiation is active infinitely often. Thus there are infinitely many stages at which work is done for the dynamic segment and the discrete segment. If $\forall u \exists v R(n, u, v)$, then for every $u$ a new witness $v$ will eventually be found. Each time one is found, the dynamic segment is densified, from which it follows that the order type $\eta$ is built. If instead $\exists u \forall v \neg R(n, u, v)$, then the dynamic segment is densified only finitely often, from which it follows that the order type FIN is built. The existence of infinitely many stages at which work is done for the discrete segment is enough for the discrete segment to have order type $\omega$ in the limit.

Additionally, the actions of $\mathcal{L}_{m}$ for $m \neq n$ do not interfere with the order type of $\mathcal{L}_{n}$. If a block $\mathcal{L}_{m}$ is attached to $\mathcal{L}_{n}$ at some point permanently, the finite amount of $\mathcal{L}_{m}$ built to that stage is successfully incorporated into the discrete segment of $\mathcal{L}_{n}$. If a block $\mathcal{L}_{m}$ is attached and detached infinitely often to $\mathcal{L}_{n}$, it is not part of the block $\mathcal{L}_{n}$. Moreover, at least each time it is detached, the discrete segment of $\mathcal{L}_{n}$ is extended. As no block $\mathcal{L}_{m}$ interferes with the singleton element segment or the dynamic segment of $\mathcal{L}_{n}$, the $\mathcal{L}_{m}$ for $m \neq n$ do not interfere with the order type of the block $\mathcal{L}_{n}$.

The claim follows, observing that $1+$ FIN $+\omega=\omega$.

Combined, we note that the claims guarantee that $\mathcal{L} \cong \sum_{a \in A} \mathcal{L}_{a}$. Claim 4.6 .3 guarantees that any wrongly started block is eventually permanently attached. Claim 4.6.4 guarantees that a block $\mathcal{L}_{n}$ is built for $a_{n}$ at the correct location, and Claim 4.6.5 guarantees that this block is of the correct order type.

This completes the proof of Lemma 4.6.

Noting that the lemma relativizes, we obtain the following corollary.

Corollary 4.7. Uniformly in

(1) an integer $k$,

(2) a $\Delta_{2 k+3}^{0}$ index for the atomic diagram $D(\mathcal{A})$ of a linear order $\mathcal{A}=(A: \prec)$ $=\left(\left\{a_{0}, a_{1}, \ldots\right\}: \prec\right)$ with distinguished least element $a_{0}$, and

(3) an index for a $\Sigma_{2 k+3}^{0}$ predicate $\exists n \forall u \exists v R(n, u, v)$, where $R(n, u, v)$ is $\Delta_{2 k+1}^{0}$, there is an index for a $\Delta_{2 k+1}^{0}$ linear order $\mathcal{L}$ such that $\mathcal{L} \cong \sum_{a \in A} \mathcal{L}_{a}$, where $\mathcal{L}_{a_{n}}=$ $1+\eta+\omega$ if $\forall u \exists v R(n, u, v)$ and $\mathcal{L}_{a_{n}}=\omega$ otherwise. 
Before continuing, we make several remarks and observations about the preceding lemma and corollary. Although Thurber's argument serves as an outline for the above proof, the argument sketched in [17] seems to be unclear: it fails to utilize chronological priorities, and allows $\omega^{*}$ chains of wrongly started blocks to exist.

By partially controlling the $\Sigma_{2 k+3}^{0}$ predicate in Corollary 4.7. we obtain control over how many copies of $\eta$ are built.

Remark 4.8. From an index of a $\Sigma_{2 k+3}^{0}$ predicate $\exists n \forall u \exists v R(n, u, v)$, where $R(n, u, v)$ is $\Delta_{2 k+1}^{0}$, we can uniformly obtain an index of a $\Sigma_{2 k+3}^{0}$ predicate $\exists n \forall u \exists v R^{\prime}(n, u, v)$ with $R^{\prime}(n, u, v)$ being $\Delta_{2 k+1}^{0}$ such that if there is an $n$ satisfying $\forall u \exists v R(n, u, v)$, then cofinitely many $n$ satisfy $\forall u \exists v R^{\prime}(n, u, v)$ (see [16]).

Thus in Corollary 4.7 we may assume that either none or cofinitely many of the $\mathcal{L}_{n}$ have order type $1+\eta+\omega$.

Therefore, under the above remark, the effect of moving from a $\Delta_{2 k+3}^{0}$ linear order to a $\Delta_{2 k+1}^{0}$ linear order as in Corollary 4.7 is that every point is replaced by a copy of $\omega$, possibly with dense intervals $\eta$ inserted after all but finitely many of the first points of the copies of $\omega$.

We continue by giving the basic construction that, when iterated, will form the crux of the construction of $\mathcal{B}_{u(S)}$ and $\mathcal{B}_{v(S)}$.

Lemma 4.9. Uniformly in

(1) an integer $n$,

(2) $\Sigma_{2 k+3}^{0}$ predicates $R_{k}$ for $0 \leq k<n$ specifying membership of $k$ in $S$, and

(3) $a \Pi_{2 n+2}^{0}$ predicate $R_{n}$ specifying membership of $n$ in $S$,

there is an index for

(a) the algebra $\mathcal{B}_{u(S \uparrow(n+1))}$ if $n \in S$ via the $\Pi_{2 n+2}^{0}$ predicate $R_{n}$, or

(b) the algebra $\mathcal{B}_{u(S \uparrow(k+1))} \oplus \mathcal{B}_{\ell \cdot \omega^{n}}$ for some integers $k<n$ and $\ell$, or the algebra $\mathcal{B}_{\ell \cdot \omega^{n}}$ for some integer $\ell$ if $n \notin S$ via the $\Pi_{2 n+2}^{0}$ predicate $R_{n}$.

Proof. Fix $n$ and an index $e$ uniformly giving the predicates $R_{k}$. We note that membership of $k$ in $S$ for $0 \leq k<n$ can be viewed as a $\Sigma_{3}^{0}$ question relative to $\Delta_{2 k+1}^{0}$ and membership of $n$ in $S$ can be viewed as a $\Pi_{2}^{0}$ question relative to $\Delta_{2 n+1}^{0}$. In order to construct an appropriate Boolean algebra, we begin by building a $\Delta_{2 n+1}^{0}$ linear order using the relativized $\Pi_{2}^{0}$ predicate. Using Corollary 4.7, we will iteratively build $\Delta_{2 n-1}^{0}, \Delta_{2 n-3}^{0}, \ldots, \Delta_{3}^{0}$, and $\Delta_{1}^{0}$ linear orders. The interval algebra of the $\Delta_{1}^{0}$ linear order so produced will be the desired Boolean algebra.

Construction: Using the $\Pi_{2}^{0}$ predicate $R_{n}$ relative to $\Delta_{2 n+1}^{0}$, we build a $\Delta_{2 n+1}^{0}$ linear order that is $1+\eta$ if the predicate $R_{n}$ holds and Fin otherwise.

Assuming we have a $\Delta_{2 k+3}^{0}$ linear order for some $k$ with $0 \leq k<n$, we describe a uniform procedure to obtain a $\Delta_{2 k+1}^{0}$ linear order. We view the $\Delta_{2 k+3}^{0}$ linear order as a $\Delta_{3}^{0}$ linear order relative to $\Delta_{2 k+1}^{0}$ and view the predicate $R_{k}$ as a $\Sigma_{3}^{0}$ predicate relative to $\Delta_{2 k+1}^{0}$. The $\Delta_{2 k+1}^{0}$ linear order is then the linear order produced by Corollary 4.7 relativized to $\Delta_{2 k+1}^{0}$.

This completes our description of the construction.

Verification: We begin by letting $\lfloor k\rfloor$ denote the greatest integer not greater than $k$ that is a member of $S$. We proceed by establishing the following claim by induction on $k$ for $k<n$. 
Claim 4.9.1. If $k \in S$, then cofinitely many of the points appearing in the $\Delta_{2 k+3}^{0}$ linear order become a copy of a suborder in the $\Delta_{1}^{0}$ linear order whose interval algebra is $\mathcal{B}_{u(S \uparrow(k+1))} \oplus \mathcal{B}_{\omega^{k+1}}$. The remaining points appearing in the $\Delta_{2 k+3}^{0}$ linear order become a copy of a suborder in the $\Delta_{1}^{0}$ linear order whose interval algebra is $\mathcal{B}_{u\left(S \uparrow\left(\left\lfloor k^{\prime}\right\rfloor+1\right)\right)} \oplus \mathcal{B}_{\omega^{k+1}}$ for some integer $k^{\prime}<k$.

If $k \notin S$, then all of the points appearing in the $\Delta_{2 k+3}^{0}$ linear order become a copy of a suborder in the $\Delta_{1}^{0}$ linear order whose interval algebra is $\mathcal{B}_{u(S \uparrow(\lfloor k\rfloor+1))} \oplus \mathcal{B}_{\omega^{k+1}}$.

Proof. If $k=0$, then the claim follows from Lemma 4.6 and Remark 4.8

Assuming the claim for all $m<k$, we show that it holds for $k$. By Corollary 4.7 and Remark 4.8, the points in the $\Delta_{2 k+3}^{0}$ linear order will become copies of $\omega^{k-\lfloor k\rfloor}$ in the $\Delta_{2\lfloor k\rfloor+3}^{0}$ linear order.

If $\lfloor k\rfloor<k$, by induction, cofinitely many of the $\Delta_{2\lfloor k\rfloor+3}^{0}$ points will have a suborder whose interval algebra is $\mathcal{B}_{u(S \uparrow(\lfloor k\rfloor+1))} \oplus \mathcal{B}_{\omega\lfloor k\rfloor+1}$ built for them, while the remaining finitely many will have a suborder whose interval algebra is $\mathcal{B}_{u\left(S \uparrow\left(\left\lfloor k^{\prime}\right\rfloor+1\right)\right)} \oplus$ $\mathcal{B}_{\omega^{\lfloor k\rfloor+1}}$ for some integer $k^{\prime}<k$ built for them. Thus each point of the $\Delta_{2 k+3}^{0}$ linear order has a suborder in the $\Delta_{1}^{0}$ linear order whose interval algebra is $\mathcal{B}_{u(S \uparrow(\lfloor k\rfloor+1))} \oplus$ $\mathcal{B}_{\omega^{k+1}}$ built for it.

If $\lfloor k\rfloor=k$, by Corollary 4.7, cofinitely many of the points in the $\Delta_{2 k+3}^{0}$ linear order will become copies of $1+\eta+\omega$ in the $\Delta_{2 k+1}^{0}$ linear order, while the remaining finitely many will become $\omega$ in the $\Delta_{2 k+1}^{0}$ linear order. Making use of the inductive hypothesis, it follows that each point in the $\Delta_{2 k+3}^{0}$ linear order has a suborder in the $\Delta_{1}^{0}$ linear order whose interval algebra is $\mathcal{B}_{u(S \uparrow(k+1))} \oplus \mathcal{B}_{\omega^{k+1}}$ built for them.

By Claim 4.9.1, at least cofinitely many of the points in the $\Delta_{2 n+1}^{0}$ linear order have a suborder in the $\Delta_{1}^{0}$ linear order whose interval algebra is $\mathcal{B}_{u(S \mid n)} \oplus \mathcal{B}_{\omega^{n}}$ built for them. If $n \in S$, then the $\Delta_{2 n+1}^{0}$ linear order is $1+\eta$, so the $\Delta_{1}^{0}$ linear order will have $\mathcal{B}_{u(S \uparrow(n+1))}$ as its interval algebra. If $n \notin S$, then the $\Delta_{2 n+1}^{0}$ linear order is FIN, so the $\Delta_{1}^{0}$ linear order will have $\mathcal{B}_{u(S \mid n)} \oplus \mathcal{B}_{\ell \cdot \omega^{n}}$ as its interval algebra.

By repeating the construction in Lemma 4.9 for increasing $n$, we are able to build computable copies of $\mathcal{B}_{u(S)}$ and $\mathcal{B}_{v(S)}$ if $S \backslash\{\omega\}$ is $\Sigma_{(2 n+3)}^{0}$.

Theorem 4.10. If $S \subseteq \omega+1$ is a set with greatest element such that $S \backslash\{\omega\}$ is $\Sigma_{(2 n+3)}^{0}$, then $\mathcal{B}_{u(S)}\left(\mathcal{B}_{v(S)}\right.$, respectively) is computable.

Proof. Let $S \subseteq \omega+1$ be a set with greatest element such that $S \backslash\{\omega\}$ is $\Sigma_{(2 n+3)}^{0}$ and let the index $e$ witness this. We build a computable linear order $\mathcal{L}_{u(S)}\left(\mathcal{L}_{v(S)}\right.$, respectively) by iterating Lemma 4.9 for increasing $n$. We note that we may assume that $S$ is infinite, else $\mathcal{B}_{u(S)}\left(\mathcal{B}_{v(S)}\right.$, respectively) is computable as $\sigma_{u(S)}\left(\sigma_{v(S)}\right.$, respectively) is computable.

The linear order $\mathcal{L}_{u(S)}\left(\mathcal{L}_{v(S)}\right.$, respectively) is constructed by building a linear order of the form $\sum_{\tau \in 2<\omega} \mathcal{L}_{\tau}$. The linear order $\mathcal{L}_{\tau}$ depends on the value of $\sigma_{u(\omega+1)}(\tau)$ $\left(\sigma_{v(\omega+1)}(\tau)\right.$, respectively) and the set $S$.

Construction: We build a linear order $\sum_{\tau \in 2<\omega} \mathcal{L}_{\tau}$, where $\mathcal{L}_{\tau}$ is as follows.

$\sigma=\omega$ : If $\sigma_{u(\omega+1)}(\tau)=\omega\left(\sigma_{v(\omega+1)}(\tau)=\omega\right.$, respectively) and $|\tau|=\ell$, the linear order $\mathcal{L}_{\tau}$ is $\omega^{\ell}$.

$\sigma=0$ : If $\sigma_{u(\omega+1)}(\tau)=0\left(\sigma_{v(\omega+1)}(\tau)=0\right.$, respectively $)$, the linear order $\mathcal{L}_{\tau}$ is the empty linear order. 
$\sigma=n$ : If $\sigma_{u(\omega+1)}(\tau)=n\left(\sigma_{v(\omega+1)}(\tau)=n\right.$, respectively $)$ with $0<n<\omega$ and $|\tau|=\ell$, we use Lemma 4.9 to build $\mathcal{L}_{\tau}$. More specifically, the $\Pi_{2 n+2}^{0}$ predicate is the $\ell^{\text {th }}$ column of the $\Sigma_{2 n+3}^{0}$ predicate giving membership of $n$ in $S$.

This completes our description of the construction.

Verification: We verify that the interval algebra of $\sum_{\tau \in 2<\omega} \mathcal{L}_{\tau}$ is $\mathcal{B}_{u(S)}\left(\mathcal{B}_{v(S)}\right.$, respectively). As $\mathcal{L}_{\tau}=\omega^{\ell}$ if $\sigma_{u(\omega+1)}(\tau)=\omega\left(\sigma_{v(\omega+1)}(\tau)=\omega\right.$, respectively) and $|\tau|=\ell$, the placement of the rank $\omega$ points is correct.

Above each rank $\omega$ point in the measure $\sigma_{u(\omega+1)}\left(\sigma_{v(\omega+1)}\right.$, respectively) are rank $n$ points for arbitrarily large $n$. For $n \in S$, a copy of $\mathcal{B}_{u(S \uparrow(n+1))}$ will appear above cofinitely many of the rank $n$ points by Lemma 4.9. As $S$ was assumed to be infinite, the placement of the rank $n$ points is correct.

It remains to verify that the points built for numbers $n \notin S$ and for the finitely many exceptional rank $n$ points for $n \in S$ do not disturb the isomorphism type of the interval algebra. In either case, by Lemma 4.9, such interval algebras will either be superatomic of small rank or $\mathcal{B}_{u(S \uparrow(k+1))}$ for some integer $k$. Because of the placement of such points, in the former case they can be thought of as contributing to the rank $\omega$ points; in the latter case they can be thought of as contributing to the rank $k$ points.

We conclude that the interval algebra of $\sum_{\tau \in 2<\omega} \mathcal{L}_{\tau}$ is $\mathcal{B}_{u(S)}\left(\mathcal{B}_{v(S)}\right.$, respectively).

\section{The LOW ${ }_{n}$-Conjecture}

As a corollary to Theorem 4.1, we resolve the low $_{n}$-Conjecture for depth zero, rank $\omega$ Boolean algebras. We recall when sets are $\operatorname{low}_{n}$ and the statement of this conjecture.

Definition 5.1. A set $A$ is $\operatorname{low}_{n}$ if $A^{(n)}=\emptyset^{(n)}$.

Conjecture 5.2 (The low $_{n}$-Conjecture). If a Boolean algebra has a copy computable in a lown set $X$ for some $n$, then it has a computable copy.

We proceed to resolving this conjecture for the depth zero, rank $\omega$ Boolean algebras.

Corollary 5.3. If a depth zero, rank $\omega$ Boolean algebra $\mathcal{B}$ has a lown presentation for some $n$, then it has a computable presentation.

Proof. Fix a low $n$ set $A$ so that $\mathcal{B}$ has an $A$-computable presentation. Relativizing Theorem 4.4 to $A$, we have that $k \in S$ if and only if a $\Sigma_{2 k+3}^{A}$ predicate holds.

For $k \geq n$, we have $\Sigma_{2 k+3}^{A}=\Sigma_{2 k+3}^{0}$ as

$$
A^{(2 k+3)}=\left(A^{(n)}\right)^{(2 k-n+3)} \equiv_{T}\left(\emptyset^{(n)}\right)^{(2 k-n+3)}=\emptyset^{(2 k+3)}
$$

and, moreover, this is uniform in $k$. Thus nonuniformly in the finite information of $S \uparrow n$, we can build a computable copy of $\mathcal{B}$.

\section{REFERENCES}

[1] C. J. Ash. A construction for recursive linear orderings. J. Symbolic Logic, 56(2):673-683, 1991. MR.1133094 (93a:03047) 
[2] C. J. Ash and J. Knight. Computable structures and the hyperarithmetical hierarchy, volume 144 of Studies in Logic and the Foundations of Mathematics. North-Holland Publishing Co., Amsterdam, 2000. MR1767842 (2001k:03090)

[3] Riccardo Camerlo and Su Gao. The completeness of the isomorphism relation for countable Boolean algebras. Trans. Amer. Math. Soc., 353(2):491-518 (electronic), 2001. MR.1804507 (2001k:03097)

[4] Rod Downey and Carl G. Jockusch. Every low Boolean algebra is isomorphic to a recursive one. Proc. Amer. Math. Soc., 122(3):871-880, 1994. MR.1203984 (95a:03044)

[5] Lawrence Feiner. Hierarchies of Boolean algebras. J. Symbolic Logic, 35:365-374, 1970. MR0282805 (44:39)

[6] László Fuchs. Infinite abelian groups. Vol. II. Academic Press, New York, 1973. Pure and Applied Mathematics. Vol. 36-II. MR0349869 (50:2362)

[7] Sergei S. Goncharov. Countable Boolean algebras and decidability. Siberian School of Algebra and Logic. Consultants Bureau, New York, 1997. MR1444819 (98h:03044b)

[8] Lutz Heindorf. Alternative characterizations of finitary and well-founded Boolean algebras. Algebra Universalis, 29(1):109-135, 1992. MR.1145559 (93b:06027)

[9] N. G. Hisamiev. Criterion for constructivizability of a direct sum of cyclic p-groups. Izv. Akad. Nauk Kazakh. SSR Ser. Fiz.-Mat., (1):51-55, 86, 1981. MR614069 (82h:20042)

[10] Irving Kaplansky. Infinite abelian groups. Revised edition. The University of Michigan Press, Ann Arbor, Mich., 1969. MR0233887 (38:2208)

[11] Jussi Ketonen. The structure of countable Boolean algebras. Ann. of Math. (2), 108(1):41-89, 1978. MR0491391 (58:10647)

[12] Julia F. Knight and Michael Stob. Computable Boolean algebras. J. Symbolic Logic, 65(4):1605-1623, 2000. MR:1812171 (2001m:03086)

[13] R. S. Pierce. Countable Boolean algebras. In Handbook of Boolean algebras, Vol. 3, pages 775-876. North-Holland, Amsterdam, 1989. MR.991610

[14] J. B. Remmel. Recursive Boolean algebras. In Handbook of Boolean algebras, Vol. 3, pages 1097-1165. North-Holland, Amsterdam, 1989. MR991614

[15] Hartley Rogers, Jr. Theory of recursive functions and effective computability. second edition, MIT Press, Cambridge, MA, 1987. MR886890 (88b:03059)

[16] Robert I. Soare. Recursively enumerable sets and degrees: A study of computable functions and computably generated sets. Perspectives in Mathematical Logic. Springer-Verlag, Berlin, 1987. MR882921 (88m:03003)

[17] John J. Thurber. Recursive and r.e. quotient Boolean algebras. Arch. Math. Logic, 33(2):121129, 1994. MR1271431 (95d:03083)

[18] John J. Thurber. Every low 2 Boolean algebra has a recursive copy. Proc. Amer. Math. Soc., 123(12):3859-3866, 1995. MR.1283564 (96b:03047)

[19] Richard Watnick. A generalization of Tennenbaum's theorem on effectively finite recursive linear orderings. J. Symbolic Logic, 49(2):563-569, 1984. MR745385 (85i:03152)

Department of Mathematics, University of Connecticut, Storrs, Connecticut 06269

E-mail address: kach@math.uconn.edu 